\title{
The Hankel Power Sum Matrix Inverse and the Bernoulli Continued Fraction
}

\author{
By J. S. Frame
}

Abstract. The $m \times m$ Hankel power sum matrix $w=V V^{T}$ (where $V$ is the $m \times n$ Vandermonde matrix) has $(i, j)$-entry $S_{i+j-2}(n)$, where $S_{p}(n)=\Sigma_{k=1}^{n} k^{p}$. In solving a statistical problem on curve fitting it was required to determine $f(m)$ so that for $n>f(m)$ all eigenvalues of $w^{-1}$ would be less than 1. It is proved, after calculating $W^{-1}$ by first factoring $W$ into easily invertible factors, that $f(m)=\left(13 m^{2}-5\right) / 8$ suffices. As by-products of the proof, close approximations are given for the Hilbert determinant, and a convergent continued fraction with $m$ th partial denominator $m^{-1}+(m+1)^{-1}$ is found for the divergent Bernoulli number series $\Sigma B_{2 k}(2 x)^{2 k}$.

1. Introduction. Defined as the product $W=V V^{T}$ of the $m \times n$ Vandermonde matrix $V=\left(j^{i-1}\right)$ with its transpose $V^{T}$, the $m \times m$ Hankel power sum matrix $W=$ $W_{m}$ has $(i, j)$ entry $S_{i+j-2}(n)$, where

$$
S_{p}=S_{p}(n)=\sum_{k=1}^{n} k^{p}=\frac{n^{p+1}}{p+1}+\frac{n^{p}}{2}+\sum_{k=1}^{[p / 2]} \frac{B_{2 k}}{2 k}\left(\begin{array}{c}
p \\
2 k-1
\end{array}\right),
$$

and where $B_{2 k}$ are the Bernoulli numbers [3], [6]:

$$
B_{2}=1 / 6, \quad B_{4}=-1 / 30, \quad B_{6}=1 / 42, \quad B_{8}=-1 / 30, \quad B_{10}=5 / 66, \ldots
$$

In solving a statistical problem involving the fitting of polynomial curves of degree $m$ to $n>m$ points, for increasing $m$ and $n$, it was required [4] to find a function $f(m)$, such that, whenever $n>f(m)$, the eigenvalues $\mu_{k}$ of $M=W^{-1}$ would all be less than 1 . We evaluate $w_{m}=\operatorname{det} W_{m}$ in Section 2 as

$$
w_{m}=\operatorname{det} w_{m}=h_{m} \prod_{i, j=1}^{m}(n+i-j),
$$

where $h_{m}$ is the determinant of the Hilbert matrix $H_{m}$, and we obtain close estimates for $h_{m}$. In Section 3 we factor $W_{m}$ as a product of easily invertible matrices of which only diagonal matrices involve $n$, and we also explicitly invert $W_{m}$ and $H_{m}$. In Section 4 we estimate the trace of $M=W^{-1}$ and find that the function

$$
f(m)=\left(13 m^{2}-5\right) / 8
$$

suffices for powers of $M$ to converge when $n>f(m)$.

As a by-product of this investigation, we find in Section 5 that the divergent

Received October 26, 1977; revised August 4, 1978.

AMS (MOS) subject classifications (1970). Primary 65 F05, $15 \mathrm{A09,} 30 \mathrm{A22}$. 0025-5718/79/0000-0078/\$04.00 
asymptotic series $B(x)$ with Bernoulli number coefficients

$$
B(x)=\sum_{k=1}^{\infty} B_{2 k}(2 x)^{2 k},
$$

related to the Laplace transform of $x \operatorname{coth} x-1$, has the convergent continued fraction expansion

$$
B(x)=\frac{x^{2}}{\mid 1+1 / 2+} \frac{x^{2} \mid}{\mid 1 / 2+1 / 3+} \frac{x^{2} \mid}{\mid 1 / 3+1 / 4+} \cdots .
$$

In fact, $B(1 / 12)=\pi^{2}-9.865$ is given with error $<2 \times 10^{-12}$ by the sixth convergent of this continued fraction.

2. The Determinants. Since $S_{0}(n)=n$ and $n(n+1) / 2$ divides $S_{k}(n)$ for $k>0$, it follows directly that $w_{m}=\operatorname{det} w_{m}$ has the algebraic factor $n^{m}(n+1)^{m-1}$. For $r$ $<m,(n-r)^{m-r}$ is also an algebraic factor of $w_{m}$, since the matrix $W_{m}(n)$ has rank $r$ and nullity $m-r$, when $n=r$ for $r=1,2, \ldots, m-1$. Since the polynomials $S_{p}(n)$. are generated by the function

$$
G(x, n)=\left(e^{x n}-1\right) /\left(1-e^{-x}\right)=\sum_{p=0}^{\infty} S_{p}(n) x^{p} / p !,
$$

we see from the identity

$$
G(x, n)+G(-x,-n-1)+1=0
$$

that

$$
S_{p}(n)+(-1)^{p} S_{p}(-n-1)+\delta_{p, 0}=0 .
$$

Hence, $w_{m}$ has the algebraic factor $(n+1+r)^{m-1-r}$. We have found $m^{2}$ linear functions of $n$ as factors of the polynomial $w_{m}(n)$ which is of degree $m^{2}$ in $n$. The remaining factor is the determinant of the leading coefficients $1 /(i+j-1)$, namely the determinant $h_{m}$ of the ill-conditioned Hilbert matrix $H_{m}$ of order $m$ [5], [7]. This proves Eq. (1.3).

If we take $m=n$ in (1.3), the Vandermonde matrix $V$ in $W=V V^{T}$ is square. Its determinant $v_{m}$ is

$$
v_{m}=\operatorname{det} V_{m}=1 ! 2 ! 3 ! \cdots(m-1) ! \equiv(m-1) ! ! \text {. }
$$

Hence by (1.3) and (2.3)

$$
h_{m}=\operatorname{det} V_{m}^{2} / \prod_{i, j=1}^{m}(m+i-j)=v_{m}^{4} / v_{2 m} .
$$

The ratio of successive $h_{m}$ 's is

$$
h_{m} / h_{m+1}=(2 m+1) !(2 m) ! /(m !)^{4}=(2 m+1)\left(\begin{array}{c}
2 m \\
m
\end{array}\right)^{2} .
$$

The following theorem gives a close approximation for $h_{m}$.

THEOREM 2.1. The determinant $h_{m}$ of the Hilbert matrix $H_{m}$ of order $m$ is 
given to 10 significant figures for $m>4$ by

$$
h_{m}=4^{-m(m-1)}(\pi / 2)^{m-1} m^{-1 / 4} \exp R_{m},
$$

where the remainder function $R_{m}$ is defined by

$$
R_{m}=\int_{0}^{\infty}\left(e^{-2 t}-e^{-2 m t}\right) \tanh ^{2}(t / 2)(4 t)^{-1} d t
$$

and is approximated to 9 decimals for $m \geqslant 5$ by

$$
\begin{aligned}
R_{m}= & 0.013081539-2^{-6} m^{-2}+2^{-8} m^{-4}-2^{-8.5} m^{-6} \\
& +2^{-8} m^{-8}-2^{-7} m^{-10}
\end{aligned}
$$

Proof. The ratio $h_{m} / h_{m+1}$ in (2.5) is related to the Wallis approximation $\pi_{m}$ for $\pi$ by

$$
\frac{\pi_{m}}{2}=\frac{2}{1} \frac{2}{3} \frac{4}{3} \frac{4}{5} \cdots \frac{2 m}{2 m-1} \frac{2 m}{2 m+1}=2^{4 m} h_{m+1} / h_{m}
$$

If we express $\ln n$ in the form

$$
\ln n=\int_{1}^{n} \frac{d s}{s}=\int_{0}^{\infty} \int_{1}^{n} e^{-s t} d s d t=\int_{0}^{\infty}\left(e^{-t}-e^{-n t}\right) t^{-1} d t
$$

then $\ln \left(\pi_{m} / 2\right)$ and its limit $\ln (\pi / 2)$ are expressible as

$$
\begin{aligned}
\ln \left(\pi_{m} / 2\right) & =\int_{0}^{\infty}\left(e^{-t}-2 e^{-2 t}+2 e^{-3 t}-2 e^{-2 m t}+e^{-(2 m+1) t}\right) t^{-1} d t \\
& =\int_{0}^{\infty}\left(e^{-t}-e^{-(2 m+1) t}\right)\left(1-e^{-t}\right)\left(1+e^{-t}\right)^{-1} t^{-1} d t
\end{aligned}
$$

$$
\begin{gathered}
\ln (\pi / 2)=\int_{0}^{\infty} e^{-t} \tanh (t / 2) t^{-1} d t \\
\ln \left(\pi / \pi_{k}\right)=\int_{0}^{\infty} e^{-(2 k+1) t} \tanh (t / 2) t^{-1} d t, \\
(\pi / 2)^{m-1} \prod_{k=1}^{m-1}\left(\pi_{k} / \pi\right)=2^{2 m(m-1)} h_{m} / h_{1} .
\end{gathered}
$$

Summing in (2.13) from $k=1$ to $m-1$ yields

$$
\begin{aligned}
\ln \left[(\pi / 2)^{m-1} 2^{-2 m(m-1)} / h_{m}\right] & =\int_{0}^{\infty}\left(e^{-2 t}-e^{-2 m t}\right)\left(e^{t / 2}+e^{-t / 2}\right)^{-2} t^{-1} d t \\
& =(1 / 4) \ln (2 m / 2)-R_{m}
\end{aligned}
$$

by (2.10), where $R_{m}$ is defined by (2.7). Equation (2.6) follows from (2.15). To obtain (2.8) we evaluate $R_{4}=.012119610988$ from (2.6) setting $h_{4}=1 / 6048000$ in (2.6). Then we compute $R_{\infty}-R_{m}$ from (2.7) by replacing $\tanh (t / 2)$ by the first five terms of its series, and set $m=4$ to get $R_{\infty}$ in (2.8). We check the tenth decimal by working from $h_{5}$ instead. This gives $\exp R_{m}$ and $h_{m}$ accurate to 10 significant figures. 
For $m=20$ we find $R_{20}=.0130425009$ and

$$
h_{20}=4.206178954 \times 10^{-226} .
$$

The matrices $H_{m}$ and $W_{m}(n)$ are ill conditioned. In fact, $W_{3}(3)$ has the eigenvalues $\lambda_{1}=113.4132, \lambda_{2}=1.564253, \lambda_{3}=.02254695$ and the conditioning ratio $\lambda_{1} / \lambda_{3}=$ 5030. So the usual computer methods for inverting $W_{m}(n)$ are unreliable [5], [7] .

3. Inversion by Factoring. To invert the ill-conditioned $m \times m$ matrix $W=$ $W_{m}(n)$ with $(i, j)$ entry $S_{i+j-2}(n)$, we first factor it into easily invertible factors, restricting the variable $n$ to diagonal matrix factors $E P=\left(\operatorname{diag} e_{i} p_{i}\right)$ and $Q=\left(\operatorname{diag} q_{j}\right)$, where

$$
e_{i}=(-1)^{i-1}, \quad p_{i}=\left(\begin{array}{c}
n+i-1 \\
n-m
\end{array}\right), \quad q_{j}=\left(\begin{array}{c}
n-j \\
n-m
\end{array}\right)
$$

We denote by $T=\left(t_{i j}\right)$ the lower Pascal triangle matrix with

$$
t_{i j}=\left(\begin{array}{c}
i-1 \\
j-1
\end{array}\right)=\left(\begin{array}{c}
i-1 \\
i-j
\end{array}\right)=(-1)^{i+j}\left(\begin{array}{c}
-j \\
i-j
\end{array}\right)
$$

We note that ETE has entries $\left({ }_{i-j}^{-j}\right)$, so

$$
(T E T E)_{i j}=\sum_{k=j}^{i}\left(\begin{array}{c}
i-1 \\
i-k
\end{array}\right)\left(\begin{array}{c}
-j \\
k-j
\end{array}\right)=\left(\begin{array}{c}
i-j-1 \\
i-j
\end{array}\right)=\delta_{i j}
$$

and $T^{-1}=E T E$. Next, we define an $m \times m$ lower triangular row stochastic matrix $A=\left(a_{i j}\right)$ that converts the integral powers in $V$ into binomial coefficients by the formula

$$
(A V)_{i k}=\sum_{r=1}^{i} a_{i r} k^{r-1}=\left(\begin{array}{c}
k+i-2 \\
i-1
\end{array}\right), \quad k=1,2, \ldots, n
$$

The $a_{i r}$ are related to Stirling numbers of the first kind, and

$$
A=\left[\begin{array}{cccccc}
1 & 0 & 0 & 0 & 0 & \ldots \\
0 & 1 & 0 & 0 & 0 & \ldots \\
0 & 1 / 2 ! & 1 / 2 ! & 0 & 0 & \ldots \\
0 & 2 / 3 ! & 3 / 3 ! & 1 / 3 ! & 0 & \ldots \\
0 & 6 / 4 ! & 11 / 4 ! & 6 / 4 ! & 1 / 4 ! & \ldots
\end{array}\right]=\left(a_{i j}\right)
$$

From (3.5) and (3.4) we obtain

$$
(A V)_{i k}=\left(\begin{array}{c}
i+k-2 \\
i-1
\end{array}\right)=\sum_{j=1}^{i}\left(\begin{array}{c}
i-1 \\
i-j
\end{array}\right)\left(\begin{array}{c}
k-1 \\
j-1
\end{array}\right)=\sum_{j=1}^{k} t_{i j}\left(\begin{array}{c}
k-1 \\
j-1
\end{array}\right) .
$$

To factor $W$ we now write 


$$
\begin{aligned}
\left(A V\left(T^{-1} A V\right)^{T}\right)_{i j} & =\sum_{k=j}^{n}\left(\begin{array}{c}
k+i-2 \\
i-1
\end{array}\right)\left(\begin{array}{c}
k-1 \\
j-1
\end{array}\right) \\
& =\left(\begin{array}{c}
i+j-2 \\
i-1
\end{array}\right) \sum_{k=j}^{n}\left(\begin{array}{c}
k+i-2 \\
j+i-2
\end{array}\right)
\end{aligned}
$$

Summing over $k$ yields

$$
\begin{aligned}
\left(A W\left(T^{-1} A\right)^{T}\right)_{i j} & =\left(\begin{array}{c}
i+j-2 \\
i-1
\end{array}\right)\left(\begin{array}{c}
n+i-1 \\
j+i-1
\end{array}\right) \\
& =p_{i}\left(\begin{array}{c}
i+j-2 \\
i-1
\end{array}\right)\left(\begin{array}{c}
m+i-1 \\
j+i-1
\end{array}\right) / q_{j}
\end{aligned}
$$

THEOREM-3.1. The inverse matrix $M=W^{-1}$ has the factorization

$$
M=W^{-1}=A^{T} E B E A, \quad B=T^{T} Q T T^{T} T P^{-1},
$$

where $E, P, Q, T, A$ are defined in (3.1), (3.2) and (3.5).

Proof. Entries of $T T^{T}$ and $T T^{T} T$ are

$$
\begin{gathered}
\left(T T^{T}\right)_{i k}=\sum_{r=1}^{i}\left(\begin{array}{c}
i-1 \\
i-r
\end{array}\right)\left(\begin{array}{c}
k-1 \\
r-1
\end{array}\right)=\left(\begin{array}{c}
i+k-2 \\
i-1
\end{array}\right) \\
\left(T T^{T} T\right)_{i j}=\sum_{k=j}^{m}\left(\begin{array}{c}
i+k-2 \\
k-1
\end{array}\right)\left(\begin{array}{c}
k-1 \\
j-1
\end{array}\right)=\left(\begin{array}{c}
i+j-2 \\
i-1
\end{array}\right) \sum_{k=j}^{m}\left(\begin{array}{c}
k+i-2 \\
j+i-2
\end{array}\right)
\end{gathered}
$$

Summing over $k$ yields

$$
\left(T T^{T} T\right)_{i j}=\left(\begin{array}{c}
i+j-2 \\
i-1
\end{array}\right)\left(\begin{array}{c}
m+i-1 \\
j+i-1
\end{array}\right)
$$

Combining (3.8) and (3.12), we have

$$
A W A^{T}=P T T^{T} T Q^{-1} T^{T} .
$$

Since the diagonal sign matrix $E$ commutes with $P$ and $Q$ but transforms $T$ and $T^{T}$ into their inverses,

$$
\left(A W A^{T}\right)^{-1}=E T^{T} Q T T^{T} T P^{-1} E=E B E
$$

and (3.9) is proved.

Equation (3.12) provides a simple method for inverting the Hilbert matrix $H$.

THEOREM 3.2. The inverse of the $m \times m$ Hilbert matrix $H=\left(h_{i j}\right)$ with $(i, j)$ entry $h_{i j}=1 /(i+j-1)$ is given by

$$
\begin{gathered}
\left(H^{-1}\right)_{i j}=d_{i} d_{i}^{\prime} h_{i j} d_{j} d_{j}^{\prime}, \\
d_{i}^{\prime}=\left(\begin{array}{c}
-m-1 \\
i-1
\end{array}\right), \quad d_{j}=m\left(\begin{array}{c}
m-1 \\
j-1
\end{array}\right) .
\end{gathered}
$$


Proof. Factoring (3.12) yields

$$
\left(E T T^{T} T\right)_{i j}=(-1)^{i-1}\left(\begin{array}{c}
m+i-1 \\
i-1
\end{array}\right) \frac{m}{i+j-1}\left(\begin{array}{c}
m-1 \\
j-1
\end{array}\right)=d_{i}^{\prime} h_{i j} d_{j}
$$

Since $E T T^{T} T$ is involutory, $D^{\prime} H D=\left(D^{\prime} H D\right)^{-1}$. Also,

$$
h_{m}=\operatorname{det} H_{m}= \pm 1 / \prod_{i=1}^{m} d_{i} d_{i}^{\prime}
$$

Although the matrix $B$ in (3.9) is symmetric, its symmetry is not obvious from formula (3.9).

THEOREM 3.3. The symmetric matrix $B=\left(b_{i j}\right)$ in (3.9) has entries expressible in terms of descending factorials $(x)_{r}=x(x-1) \cdots(x-r+1)$ as follows:

$$
b_{i j}=\sum_{r \geqslant i+j-1} \frac{(m+i-1)_{r}(m+j-1)_{r}}{(n-m+r)_{r} r !}\left(\begin{array}{c}
r-1 \\
i-1, j-1
\end{array}\right),
$$

where $\left(\begin{array}{c}r-1 \\ i-1, j-1\end{array}\right)$ is the trinomial coefficient

$$
\left(\begin{array}{c}
r-1 \\
i+j-2
\end{array}\right)\left(\begin{array}{c}
i+j-2 \\
i-1
\end{array}\right)
$$

Proof. To transform $B$ in (3.9) we evaluate

$$
\begin{aligned}
& \left(T^{T} Q T T^{T}\right)_{i s}=\sum_{r=i}^{m}\left(\begin{array}{c}
r-1 \\
i-1
\end{array}\right)\left(\begin{array}{c}
n-r \\
n-m
\end{array}\right)\left(\begin{array}{c}
r+s-2 \\
r-1
\end{array}\right) \\
& =\left(\begin{array}{c}
i+s-2 \\
i-1
\end{array}\right) \sum_{r=i}^{m}\left(\begin{array}{l}
r+s-2 \\
i+s-2
\end{array}\right)\left(\begin{array}{c}
n-r \\
n-m
\end{array}\right) \\
& =\left(\begin{array}{c}
i+s-2 \\
i-1
\end{array}\right)\left(\begin{array}{c}
n+s-1 \\
m-i
\end{array}\right) \text {, } \\
& (B P)_{i j}=\sum_{s=j}^{m}\left(\begin{array}{c}
i+s-2 \\
i-1
\end{array}\right)\left(\begin{array}{c}
n+s-1 \\
m-i
\end{array}\right)\left(\begin{array}{c}
s-1 \\
j-1
\end{array}\right) \\
& =\left(\begin{array}{c}
i+j-2 \\
i-1
\end{array}\right) \sum_{s=j}^{m}\left(\begin{array}{c}
i+s-2 \\
s-j
\end{array}\right) \sum_{r \geqslant i+j-1}\left(\begin{array}{c}
s-j \\
r-i-j+1
\end{array}\right)\left(\begin{array}{c}
n+j-1 \\
m+j-1-r
\end{array}\right) \\
& =\sum_{r \geqslant i+j-1} \sum_{s=j}^{m}\left(\begin{array}{c}
i+s-2 \\
r-1
\end{array}\right)\left(\begin{array}{c}
r-1 \\
i-1, j-1
\end{array}\right)\left(\begin{array}{c}
n+j-1 \\
n-m+r
\end{array}\right) .
\end{aligned}
$$

Summing over $s$ and dividing by $p_{j}$, we have

$$
b_{i j}=\sum_{r \geqslant i+j-1}\left(\begin{array}{c}
m+i-1 \\
r
\end{array}\right)\left(\begin{array}{c}
r-1 \\
i-1, j-1
\end{array}\right)\left(\begin{array}{c}
m+j-1 \\
r
\end{array}\right) /\left(\begin{array}{c}
n-m+r \\
r
\end{array}\right) \text {. }
$$

Writing $(x)_{r}=r !\left(\begin{array}{l}x \\ r\end{array}\right)$, Eq. (3.21) becomes (3.18). 
To conserve space in displaying the symmetric matrices $M_{m}(n)=W_{m}^{-1}(n)$ we show the upper half of $M_{3}$ and the lower half of $M_{4}$.

(3.22)

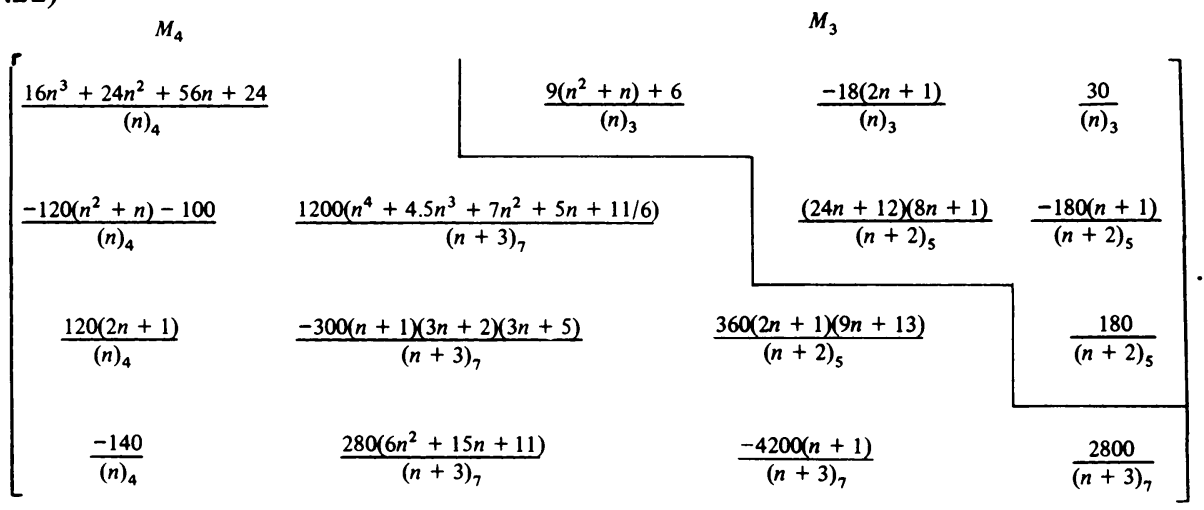

4. Estimation of $\operatorname{tr} M$. Since $W$ and $M$ are positive definite for $n>m$, all eigenvalues $\mu_{k}$ of $M$ will satisfy $\mu_{k}<1$ if $\operatorname{tr} M \leqslant 1, m>1$. For $A$ in (3.5) and $e_{i}=(-1)^{i-1}$, the first and last diagonal entries of $M=M_{m}$ are $b_{11}$ and $b_{m m} /((m-1) !)^{2}$. Numerical computation shows that the maximum $n$ for which $\operatorname{det}\left(W_{m}(n)-I\right)=0$ are given for $m=1,2,3,4$ by

$$
(m, n)=(1,1),(2,5.82090),(3,13.3776),(4,24.24453) .
$$

The parabola through the first three points is

$$
n=g(m)=1.5679 m^{2}-.0828 m-.4851,
$$

and we find $g(4)=24.270>24.24453$. A slightly higher value than (4.2) will be required for $\operatorname{tr} M \leqslant 1$. We first estimate the dominant diagonal entry $b_{11}$ of $M$.

$$
\left(\begin{array}{l}
n \\
m
\end{array}\right) b_{11}=\sum_{r=1}^{m}\left(\begin{array}{l}
m \\
r
\end{array}\right)^{2}\left(\begin{array}{l}
n \\
m
\end{array}\right) /\left(\begin{array}{c}
n-m+r \\
r
\end{array}\right)=\sum_{r=1}^{m}\left(\begin{array}{l}
m \\
r
\end{array}\right)\left(\begin{array}{c}
n \\
m-r
\end{array}\right)
$$

$$
=\left(\begin{array}{c}
n+m \\
m
\end{array}\right)-\left(\begin{array}{l}
n \\
m
\end{array}\right) \text {. }
$$

$$
\begin{aligned}
& \text { (4.4) } 1+b_{11}=\left(\begin{array}{c}
n+m \\
m
\end{array}\right) /\left(\begin{array}{l}
n \\
m
\end{array}\right)=\prod_{k=1}^{m} \frac{2 n+1+(2 k-1)}{2 n+1-(2 k-1)} \\
& \text { (4.5a) } \ln \left(1+b_{11}\right)=\sum_{k=1}^{m} \ln \frac{1+(2 k-1) /(2 n+1)}{1-(2 k-1) /(2 n+1)}=\sum_{r=1}^{\infty} \frac{\theta(m, r)}{(2 r-1)(2 n+1)^{2 r-1}}
\end{aligned}
$$

where

$$
\theta(m, r)=\sum_{k=1}^{m} 2(2 k-1)^{2 r-1}<\int_{0}^{2 m} x^{2 r-1} d x=(2 m)^{2 r} / 2 r
$$

We now assume the inequalities $n>f(m)$ in (1.4).

THeOREM 4.1. The matrix $M=W_{m}^{-1}(n)$ has trace $<1$ if

$$
n>1.625 m^{2}-.625 \text { and } m \geqslant 5 \text {. }
$$


Proof. If (4.6) is satisfied for $m=5$, then $n \geqslant 40$, and

$$
b_{11} \leqslant(45)_{5} /(40)_{5}-1=62639 / 73112=.856754 .
$$

If (4.6) is satisfied for $m \geqslant 6$, then $(2 n+1) / 2 m^{2}>1.6215$ and

$$
\ln \left(1+b_{11}\right)<\frac{2 m^{2}}{2 n+1} \sum_{r=1}^{\infty}\left(\frac{2 m}{2 n+1}\right)^{2 r-2} / r(2 r-1)
$$

$$
<\frac{1}{1.6215} \sum_{r=1}^{\infty}\left(\frac{1}{9.729}\right)^{2 r-2} / r(2 r-1)<.62777 \text {, }
$$

$$
b_{11}<.8734 \text { for } m \geqslant 6, n>\left(13 m^{2}-5\right) / 8 .
$$

The rest of $\operatorname{tr} M$ is given by

$$
\operatorname{tr} M-b_{11}=\sum_{k=2}^{m} \sum_{i, j=k}^{m} a_{i k}(-1)^{i} b_{i j}(-1)^{j} a_{j k} .
$$

We replace $i, j, r$ by $i+1, j+1, r+2$ and write

(4.10) $b_{i+1, j+1}=\sum_{k=1}^{m-1} y_{i j}^{(r)}, \quad y_{i j}^{(r)}=\frac{(m+i)_{r+2}(m+j)_{r+2}}{(n-m+r+2)_{r+2}(r+2) !}\left(\begin{array}{c}r+1 \\ i, j\end{array}\right)=y_{j i}^{(r)}$.

Then

(4.11) $\operatorname{tr} M-b_{11}=y_{11}^{(1)} \sum_{r=1}^{2 m-1} \varphi_{m n}(r), \quad \varphi_{m n}(r)=\sum_{i+j=2}^{r+1} c_{i j} y_{i j}^{(r)} / y_{11}^{(1)}$,

where the entries of the $(m-1) \times(m-1)$ matrix $C=\left(c_{i j}\right)$ are

$$
c_{i j}=(-1)^{i+j} \sum_{k=1}^{m-1} a_{i+1, k} a_{j+1, k}=c_{j i} \text {, }
$$

$(4.12 b) C=\frac{1}{720}\left[\begin{array}{rrrrrr}720 & -360 & 240 & -180 & 144 & \ldots \\ -360 & 360 & -300 & 255 & -222 & \ldots \\ 240 & -300 & 280 & -255 & 233 & \ldots \\ -180 & 255 & -255 & 242.5 & -228.5 & \ldots \\ 144 & -222 & 233 & -228.5 & 220.1 & \ldots \\ . & . & . & . & . & \ldots\end{array}\right]$.

The dominant term $y_{11}^{(1)}$ satisfies

$$
y_{11}^{(1)}<\frac{(m+1)_{3}(m+1)_{3} / 3}{\left(13 m^{2} / 8-m+19 / 8\right)_{3}} \leqslant \frac{6_{3} 6_{3} / 3}{(38)_{3}}=\frac{200}{2109}<.094832,
$$

since the rational function decreases for $5 \leqslant m$. The function $\varphi_{m n}(1)$ is 1 , but for $r$ $>1$, then $\varphi_{m n}(r)$ in (4.11) are bounded by rational functions which increase for 
$m \geqslant 5$, and which we replace by their limits as $m \rightarrow \infty$.

$$
\varphi_{m n}(2)=\left(y_{11}^{(2)}-y_{12}^{(2)}\right) / y_{11}^{(1)}=3(m-2)(m-6) /\left(13 m^{2}-8 m+27\right)
$$

$$
\begin{aligned}
& <3 / 13=.23077 \\
\varphi_{m n}(3)= & \left(y_{11}^{(3)}-y_{12}^{(3)}+2 y_{13}^{(3)} / 3+y_{22}^{(3)} / 2\right) / y_{11}^{(1)} \\
& <17\left(m^{2}-6 m+32\right)(m-2)(m-2.4) /(120)\left(13 m^{2} / 8-m+35 / 8\right)_{2} \\
& <(17 / 120)(8 / 13)^{2}=.05365 .
\end{aligned}
$$

Similar calculations yield

$$
\varphi_{m n}(4)<(1 / 32)(8 / 13)^{3}=.00728 .
$$

Since the coefficients of $(8 / 13)^{r-1}$ in $\varphi_{m n}(r)$ decrease as $r$ increases, the remaining sum of $\varphi_{m n}(r)$ is $<2.6 \varphi_{m n}(4)$. Hence, (4.11) implies

$$
\begin{aligned}
\operatorname{tr} M & <.8734+.095(1.23077+.05365+3.6(.00728)) \\
& <.8734+.095(1.3107)<.998<1 .
\end{aligned}
$$

This proves Theorem 4.1. We check directly for $m=2,3,4$ that

$$
\begin{aligned}
& \operatorname{tr} M_{2}(6)=97 / 105, \quad \operatorname{tr} M_{3}(14)=.95+1 / 7280 \\
& \operatorname{tr} M_{4}(25)=.87755+.09359+.0073+.0000005<.9719
\end{aligned}
$$

This proves the parabolic bound $n>f(m)=\left(13 m^{2}-5\right) / 8$ to be sufficient for $\operatorname{tr} M$ $<1$. Although some bound between this and $n>g(m)$ in (4.2) might also suffice for all $n$, the tight inequality (4.15) indicates that it would be difficult to prove.

5. The Bernoulli Continued Fraction. The entries $S_{i+j-2}(n) / n$ of the matrix $W_{m}(n) / n$ have as constant terms the Bernoulli numbers $B_{i+j-2}$ given in (1.2). The limit as $n \rightarrow 0$ of the leading principal minor of $W_{m}(n) / n$ is the determinant $b_{m-1}^{*}$ of order $m-1$ expressible as

$$
b_{m-1}^{*}=\operatorname{det}\left(B_{i+j}\right)=\lim _{n \rightarrow 0}\left(n b_{11}\right)\left(n^{-m} w_{m}(n)\right) .
$$

Recalling $b_{11}$ from (4.3), $w_{m}(n)$ from (1.3), $v_{m}$ from (2.3) and $h_{m}$ from (2.4), we have

$$
\begin{gathered}
\lim _{n=0} n b_{11}=\left(\begin{array}{l}
m \\
m
\end{array}\right) m /\left(\begin{array}{c}
-1 \\
m-1
\end{array}\right)=(-1)^{m-1} m, \\
\lim _{n=0} n^{-m} w_{m}(n)=h_{m} v_{m}^{2}(-1)^{m(m-1) / 2}
\end{gathered}
$$




$$
b_{m-1}^{*}=(-1)^{(m-1)(m-2) / 2} m v_{m}^{6} / v_{2 m} \text {, }
$$

$$
b_{m}^{*} / b_{m-1}^{*}=(-1)^{m-1}(m-1) !(m !)^{4}(m+1) ! /(2 m) !(2 m+1) ! \text {. }
$$

Since $B_{i+j}=0$ for odd $i+j$, we can rearrange rows and columns of the matrix $\left(B_{i+j}\right)$ so the odd numbered ones precede the even numbered ones, and thus factor $b_{m-1}^{*}$ as the product $d_{m-1} d_{m-2}$ of two determinants, where

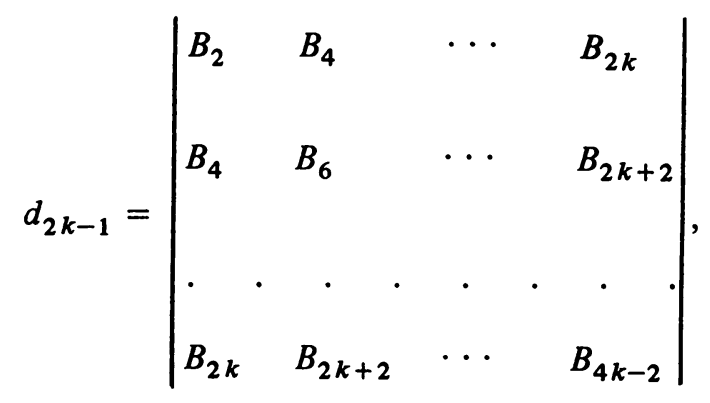

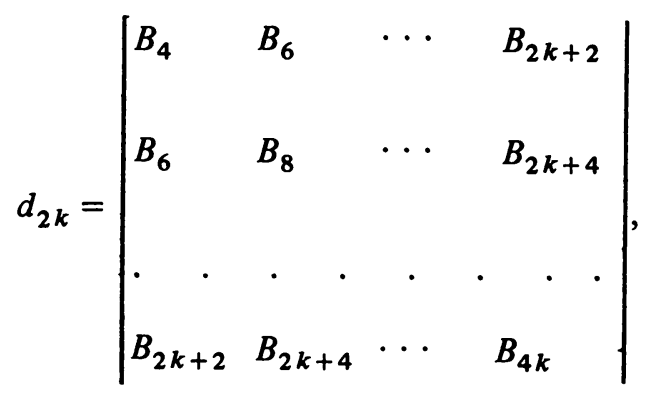

$$
\begin{aligned}
-d_{m-3} d_{m} / d_{m-1} d_{m-2} & =(m-1) m^{4}(m+1) /(2 m-1)(2 m)^{2}(2 m+1) \\
& =(1 / 4)((m-1) m /(2 m-1))(m(m+1) /(2 m+1)) .
\end{aligned}
$$

THEOREM 5.1. The divergent asymptotic alternating series

$$
B(x)=\sum_{k=1}^{\infty} B_{2 k}(2 x)^{2 k}=4 x^{2} / 6-16 x^{4} / 30+64 x^{6} / 42 \cdots
$$

has the convergent continued fraction expansion (1.6).

Proof. By the general theory of continued fractions [2] , [9], if a formal power series (5.9) with arbitrary coefficients $B_{2 k}$ is expanded into continued fractions of the form

$$
\frac{a_{1}(2 x)^{2} \mid}{1+} \frac{a_{2}(2 x)^{2} \mid}{1+} \cdots=\frac{x^{2} / c_{0} \mid}{\mid c_{1}+} \frac{x^{2} \mid}{\mid c_{2}+} \frac{x^{2} \mid}{\mid c_{3}+} \cdots
$$

and if the $d_{k}$ 's are defined by (5.6), then 


$$
a_{m}=1 / 4 c_{m-1} c_{m}=-d_{m-3} d_{m} / d_{m-2} d_{m-1}, \quad m \geqslant 1
$$

For the Bernoulli series Eqs. (5.5) and (5.11) imply

$$
c_{m}=(m(m+1) /(2 m+1))^{-1}=1 / m+1 /(m+1), \quad m \geqslant 1,
$$

while the condition $1 / c_{0} c_{1}=4 B_{1}=2 / 3$ implies $c_{0}=1$. Since $\Sigma c_{m}$ is divergent, the continued fraction (1.6) converges, and Theorem 5.1 is proved.

We can apply this continued fraction to approximate $\pi^{2}$. It would require about a billion terms of the series $\Sigma_{1}^{\infty}\left(1 / k^{2}\right)$ to approximate $\pi^{2} / 6$ to nine decimals. But the Euler-Maclaurin summation formula gives the remainder after 5 terms by the expression

$$
\int_{6}^{\infty} x^{-2} d x+1 / 2 \cdot 6^{2}+\sum_{k=1}^{\infty} B_{2 k}(1 / 6)^{2 k+1}
$$

This alternating series diverges, with minimum remainder of about $10^{-15}$ after the 19 th term. Using the convergent continued fraction instead, we have

$$
\begin{aligned}
\pi^{2} & =6(1+1 / 4+1 / 9+1 / 16+1 / 25+1 / 6+1 / 72)+B(1 / 12) \\
& =9.865+\frac{12^{-2}}{\mid 1+1 / 2+} \frac{12^{-2}}{\mid 1 / 2+1 / 3+} \frac{12^{-2} \mid}{\mid 1 / 3+1 / 4+},
\end{aligned}
$$

$$
\begin{aligned}
\pi^{2} & =9.865 \\
+ & \frac{1 / 12}{\mid 12+6+} \frac{1}{\mid 6+4+} \frac{1}{\mid 4+3+} \frac{1}{\mid 3+2.4+} \frac{1}{\mid 2.4+2+} \frac{1}{\mid 2+r},
\end{aligned}
$$

where the sixth convergent with $r=12 / 7$ has an error about $10^{-12}$, and the tenth convergent (which changes this $r$ to 1.9976 ) has an error less than $10^{-15}$, giving $\pi^{2}=$ 9.869604401089359.

The function $s^{-1} B\left(s^{-1}\right)$ is the Laplace transform of $x \operatorname{coth} x-1$.

Continued fractions for the Laplace transforms of $\tanh x, \operatorname{sech} x$ and $x \operatorname{csch} x \operatorname{can}$ also be obtained by similar methods, but have already been derived by Stieltjes [8] and others, and are listed by Wall $[9$, p. 369]. The author has not found the continued fraction (1.6) in the literature, nor the determinantal formula (5.4) which evaluates the first principal $m \times m$ minor $b_{m}^{*}=\left|B_{i+j}\right|, i, j=1, \ldots$, (omitting $B_{0}$ and $B_{1}$ ) of the determinant $\left|B_{i+j-2}\right|$ of order $m+1$ called $\Delta_{m}(B)$ by Al-Salam and Carlitz [1, p. 93, (3.1)] which in the notation of (2.3) becomes

$$
\Delta_{m}(B)=(-1)^{m(m+1) / 2}(m ! !)^{6} /(2 m+1) ! ! .
$$

Comparing (5.16) with (5.4) for order $m$, we have

$$
\left|B_{i+j}\right|_{m}=(-1)^{m}(m+1)\left|B_{i+j-2}\right|_{m+1} .
$$


1. W. A. AL-SALAM \& L. CARLITZ, "Some determinants of Bernoulli, Euler, and related numbers," Portugal. Math., v. 18, 1959, pp. 91-99.

2. J. S. FRAME, "The solution of equations by continued fractions," Amer. Math. Month$l y$, v. 60, 1953, pp. 293-305.

3. J. S. FRAME, "Bernoulli numbers modulo 27000," Amer. Math. Monthly, v. 68, 1961, pp. 87-95.

4. D. C. GILLILAND \& JAMES HANNAN, Detection of Singularities in the Countable General Linear Model, Department of Statistics, Michigan State University, RM-217, DCG-8, JH-10, Aug. 1971.

5. E. ISAACSON \& H. B. KELLER, Analysis of Numerical Methods, Wiley, New York, 1966, pp. 196, 217-218.

6. N. E. NÖRLUND, Vorlesung über Differenzenrechnung, Springer, Berlin, 1924, p. 18.

7. G. M. PHILLIPS \& P. J. TAYLOR, Theory and Application of Numerical Analysis, Academic Press, New York, 1973, pp. 91, 246.

8. T. J. STIELTJES, "Sur quelques intégrales définies et leur dévéloppement en fractions continues," Oeuvres Completes, vol. 2, P. Noordhoff, Groningen, 1918, pp. 378-391.

9. H. S. WALL, Analytic Theory of Continued Fractions, D. Van Nostrand, Princeton, N. J., 1948, pp. 369-376. 